\title{
Enhanced Tunneling Technique for Flow-Based Fast Handover in Proxy Mobile Ipv6 Networks
}

\author{
Yunes Abdussalam Amgahd \\ Student, Ph.D. Scholar \\ Department of computer Science and Engineering \\ Sam Higginbottom institute of agriculture technology and \\ sciences \\ Allahabad U.P. (211007) India
}

\author{
Raghav Yadav \\ Assistant Professor \\ Department of computer Science and Engineering \\ Sam Higginbottom institute of agriculture technology and \\ sciences \\ Allahabad U.P. (211007) India
}

\begin{abstract}
In the Mobile IPv6 network, each node is highly mobile and handoff is a very common process. When not processed efficiently, the handoff process may result in large amount of packet loss. If the handover process is performed without appropriate connection verification and through specified tunnels, then this may result in inappropriate traffic flow since the required traffic redirection may not happen in this case. To overcome these issues, we propose to develop an Enhanced Tunneling Technique for Flow-based Fast Handover in Proxy Mobile IPv6 Networks. In this technique, the packets are buffered to minimize the packet loss during handover and then the Flow based Fast Handover techique is employed to ensure that the traffic is redirected to the new subnet after handover process. Thus ensuring efficient network operation.
\end{abstract}

\section{INTRODUCTION}

\section{A. Mobile IPV6 Networks}

The Mobile IPv6(MIPv6) is an IETF standard that allows mobile device users to move from their Home Network to another while maintaining a permanent IP address, thereby ensuring location transparency (similar to a Distributed System Environment). This facility allows for seamless and continuous internet connectivity. However, the reason for the lack of popularity in deploying Mobile IPv6 is due to poor handoff latency and other drawbacks leading to packet loss and poor performance for live audio and video streamingbased applications. MIPv6 has not seen commercial interest because it requires the mobile equipment itself to participate in the mobility process and this reduces the battery life of the mobile equipment. While MIPv6 manages mobility for a single host, the Network Mobility Basic Support Protocol (NEMO BSP) Although host-based Mobile IPv6 protocols have been studied extensively, a different approach where the entire network moves, known as Network Mobility (NEMO) is of great interest in the manages mobility for an entire network [1].

Mobility in IPv6 networks has evolved remarkably compared to Mobile IPv4 protocol. Mobile IPv6 (MIPv6) enables transparent routing of IPv6 packets to Mobile Nodes (MNs) from Correspondent Nodes (CNs). The mobility is made possible by using a Home Agent (HA) and a local Careof-Address (CoA). Unfortunately it is still unsolved how to minimize the handover time between two logical subnets so that the outtime is as short as possible. [2].
Proxy Mobile IPv6 (PMIPv6) provides IP mobility to a mobile node by the proxy mobility agent called a Local Mobility Anchor and a Mobile Access Gateway without requiring mobile node's participation in any mobility-related signaling. The route optimization for PMIPv6 is realized by using the direct tunnel established between the MAGs, to which mobile nodes are attached [3].

Bidirectional tunneling is presented in Mobile IPv6 in which MN and HA are connected to each other via a tunnel, so signaling is required to construct a tunnel between $\mathrm{MN}$ and $\mathrm{CN}$ [4].

\section{RELATED WORKS}

In Flow based Fast Handover for MIPv6 (FFHMIPv6) [2], each traffic flow can be identified and redirected to a new location using the IPv6 Flow Label. It allows the reception of packets simultaneously with the BU registration process, thus minimizing the delay experienced in the handover.

A right-time path switching method has been proposed [3] for providing PMIPv6 route optimization. By using signaling messages, this method initiates the path switch when the optimized path is ready. Out-of-sequence packets are preventing by this feature. The disruption duration is reduced in the route optimization procedure. By using actual PCs, this procedure is evaluated in an experimental test-bed. Results show that this method prevents out-of-sequence packets, whereas the baseline route optimization procedure causes them. During the route optimization procedure, this method has performance improvement in TCP throughput or seamless continuity of real-time applications. Communication disruption duration, delay gap, and number of out-of-sequence packets are the performance metrics used. However, it may be affected by malicious nodes in the network [3].

An improved tunneling-based route optimization mechanism is proposed [4] to reduce the packet overhead. The tunnel manager is changed and binding update messages are altered for maintaining the compatibility with standard mechanisms. This mechanism shows the reduced packet overhead when compared to bidirectional tunneling, route optimization, and tunneling-based route optimization. In mobile IP communication, more data can be transmitted via network because of less overhead for each packet. Overhead and delay are the performance metrics used. However, the 
total delay is same as that of the bidirectional tunneling, route optimization, and tunneling-based route optimization mechanisms; hence, it must be reduced.

Khaled Zeraoulia et al [5] have introduced a novel mobility management strategy for mobile IP networks, in which they developed a seamless handover scheme called SHMIPv6 (Seamless Multimedia handoff for hierarchical Mobile IPv6). By integrating MAC and Network layer handovers efficiently, SHMIPV6 can significantly reduce the system signaling cost and handover delay.

Ali Safa Sadiq et al [6] have proposed an Advanced Mobility Handover scheme (AMH) in this paper for seamless mobility in MIPv6-based wireless networks. In the proposed scheme, the mobile node utilizes a unique home IPv6 address developed to maintain communication with other corresponding nodes without a care-of-address during the roaming process. TheIPv6 address for each MN during the first round of AMH process is uniquely identified by HA using the developed MN-ID field as a global permanent, which is identifying uniquely the IPv6 address of MN. Moreover, a temporary MN-ID is generated by access point each time an $\mathrm{MN}$ is associated with a particular AP and temporarily saved in a developed table inside the AP. When employing the AMH scheme, the handover process in the network layer is performed prior to its default time. That is, the mobility handover process in the network layer is tackled by a trigger developed AMH message to the next access point. Thus, a mobile node keeps communicating with the current access point while the network layer handover is executed by the next access point.

Mohit Bagde and P. Sankar [7] have proposed a method for improving the mobility using the fast handover of Mobile IPv6 (FMIPv6) and the Reverse Routing Header Protocol (RRH). The (RRH) protocol is employed is to record the route through which the packet travels from the Correspondent Node to the Mobile Node.

\section{ENHANCED TUNNELING TECHNIQUE FOR FLOW-BASED FAST HANDOVER IN PROXY MOBILE IPV6 NETWORKS}

\section{A. Overview}

In our previous paper, we propose to design a tunnelingbased routing and handover decision model for Proxy Mobile IPv6 networks. A tunneling based route optimization is applied in the architecture of PMIPv6 followed by a handover decision model for network selection based on various priorities of traffic classes.

As an extension to this work, we propose an Enhanced Tunneling Technique for Flow based Fast Handover in Proxy Mobile IPv6 networks.

In this technique, first the flow based fast handover (FFH) technique [2] is implemented. Then the loss-free buffering process [5] is applied to minimize the packet loss during handover process by enabling the temporary storage of the tunnel. 


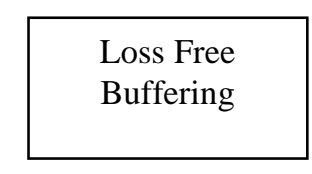

FFM Technique

Fig. 1. Block Diagram

\section{B. Loss Free Buffering Process}

During the handover process, the connection between the mobile node being handovered and the access router gets broken and the mobile node gets connected to a new access router belonging to the new subnet. During the period from when the mobile node gets disconnected from the old access router and till it gets connected to a new access router, there are chances for packets to be lost from the mobile node [5]. To avoid or to reduce the packet loss occuring during handoff process, the packets can be temporarily stored in a buffer. This buffering process to reduce packet loss is described in algorithm 1 .

\section{Algorithm 1}

\section{Notations:}

$\begin{array}{lll}\text { 1. MN } & : & \text { Mobile Node } \\ \text { 2. SS } & : & \text { Signal Strength } \\ \text { 3. } \mathrm{Sec}_{\mathrm{Th}} & : & \text { Security Threshold } \\ \text { 4. } \mathrm{SS}_{\mathrm{Th}} & : & \text { Signal Strength Threshold } \\ \text { 5. } \mathrm{R}_{\text {old }} & : & \text { old access router } \\ \text { 6. } \mathrm{R}_{\text {new }} & : & \text { new access router }\end{array}$

1) Before the handover process, a Handoff_Initiate message is broadcasted in the network.

2) After boadcasting the message, the $M N$ defines the $S_{T h}$ for the packets.

3) Then the MN keeps monitoring the SS of the packets.

4) When $\operatorname{Sec}_{T h}=\mathrm{SS}$, then the $\mathrm{MN}$ sends an Buffering_initiation message to the $\mathrm{R}_{\text {old }}$.

5) When the $R_{\text {old }}$ receives this message, it initiates storing the packets in a buffer.

6) While buffering, the $\mathrm{R}_{\text {old }}$ also sends copy of the packets to the MN.

7) When the $\mathrm{SS}<\mathrm{SS}_{\mathrm{Th}}$, the connection between the $\mathrm{MN}$ and $R_{\text {old }}$ is disconnected, and so, the $R_{\text {old }}$ stops sending the copy of the packets to the MN.

8) When the $M N$ gets connected to a $R_{\text {new }}$, the $R_{\text {old }}$ stops storing the packets in the buffer.

9) Then the $\mathrm{MN}$ operates with aid of the $\mathrm{R}_{\text {new. }}$.

In this way, the packet loss is minimized during handoff in the network by storing the packets in the buffer.

\section{Flow based Fast Handover (FFH) technique [2]}

The Flow based Fast Handover (FFH) technique is employed to handle the handover process in an efficient way. When the Mobile Node moves from one subnet to another, the 
router controlling the traffic flow towards the mobile node also changes. This information about the new subnet should be registered inorder to ensure that the traffic flow is redirected in the appropriate path.

The process of FFH performing the handoff technique along with the creating of MIPv6 tunnel is described in algorithm 2 .

\section{Algorithm 2}

\section{Notations:}

1. $\mathrm{MN}$

2. CoA

3. $\mathrm{HA}$

4. $\mathrm{CN}$

5. FFH

6. MIPv6

7. BU

\section{Algorithm:}

1) When a $\mathrm{MN}$ moves from one logical subnet to another, it recieves a new CoA.

2) On receiving the $\mathrm{CoA}$, a Flow Label of the prior mobile connection and a Hop by Hop frame containing the address of the $\mathrm{HA}$ and $\mathrm{CN}$ are added into $\mathrm{BU}$ register message.

3) The BU register message is sent to the immediate crossover router.

4) The Hop by Hop frame contains a new FFHMIPv6 identifier which indicates that IPv6 tunnel has not yet created.

5) Every router that receives the BU register message, checks the Hop by Hop frame.

6) If a router detects the FFHMIPv6 identifier, then it handles the register message to create a tunnel.

7) $\mathrm{Ob}$ the basis of the traffic information present in the messge, the router determines the connection.

8) Then an BU Ack message is sent to the MN's new CoA inorder to inform it about the crossover router.

9) Next an IPv6 tunnel is developed between the crossover router and $\mathrm{MN}$, and the traffic is redirected through the tunnel.

10)In the Hop by Hop frame, the FFHMIPv6 identifier is set to 1 to indicate that the tunnel is already created and traffic can directly be transferred through the tunnel.

11)Then the BU register message is forwarded to HA and $\mathrm{CN}$.

12) Similarly, a BU Ack message is sent by $\mathrm{HA}$ and $\mathrm{CN}$ after verifying the connection.

13)Then a IPv6 tunnel is created between the new CoA and HA and then similarly between new CoA and CN.

14)Finally all the traffic flowing towards the old CoA are encapsulated to a new IPv6 packet and redirected towards the new CoA.
So, all the traffic are redirected after the handover process. This is performed after ensuring the connection and thus ensuring packet security. To assure secure traffic flow after handover, the traffic flow is encapsulated and transmitted through the IPv6 tunnel. Thus assuring safe network operation.

\section{Simulation Results}

\section{A. Simulation Parameters}

We use NS2 to simulate our proposed Enhanced Tunneling technique for Flow based Fast Handover (ETFH) in Proxy Mobile IPv6 Networks. The area size is 600 meter x 600 meter square region for 50 seconds simulation time.

Our simulation settings and parameters are summarized in table 1

TABLE I. SimULATION PARAMETERS

\begin{tabular}{|l|l|}
\hline No. of Nodes & 16 \\
\hline Area & 600 X 600m \\
\hline MAC & 802.11 \\
\hline Simulation Time & $50 \mathrm{sec}$ \\
\hline Traffic Source & CBR and Exponential \\
\hline Rate & $50,100,150,200$ and $250 \mathrm{~Kb}$ \\
\hline Propagation & TwoRayGround \\
\hline Antenna & OmniAntenna \\
\hline Psize & 512 \\
\hline
\end{tabular}

B. Performance Metrics

We evaluate performance of the mainly according to the following parameters. We compare the Flow based Fast Handover (FFH) technique [2] with the proposed ETFFH.

Average Packet Delivery Ratio: It is the ratio of the number of packets received successfully and the total number of packets transmitted.

Delay: It is the time taken by the data packets to reach the destination.

Packet Drop: It is the number of packets dropped during the data transmission.

\section{Results \& Analysis}

The simulation results are presented in the next section.

1) Varying the Rate

In our first experiment we vary the transmission rate as 50 , $100,150,200$ and $250 \mathrm{~Kb}$ for both CBR and Exponential (EXP) traffic flows. 


\section{Case-1(CBR)}

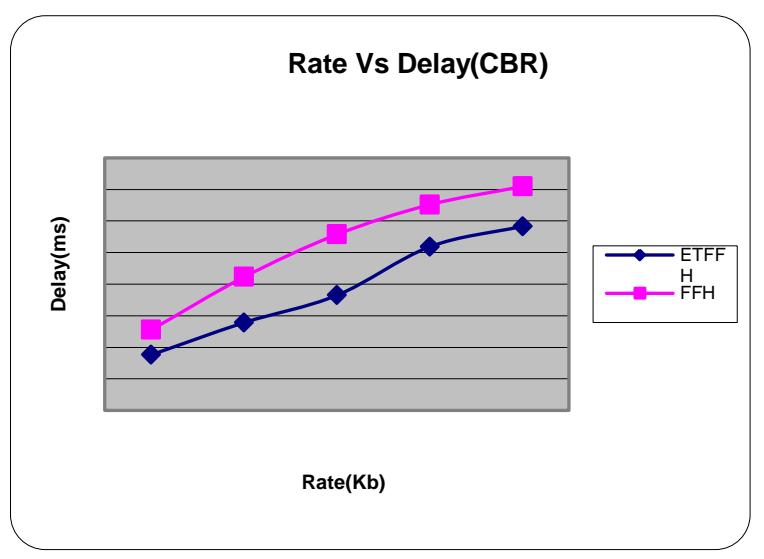

Fig. 2. Rate Vs Delay

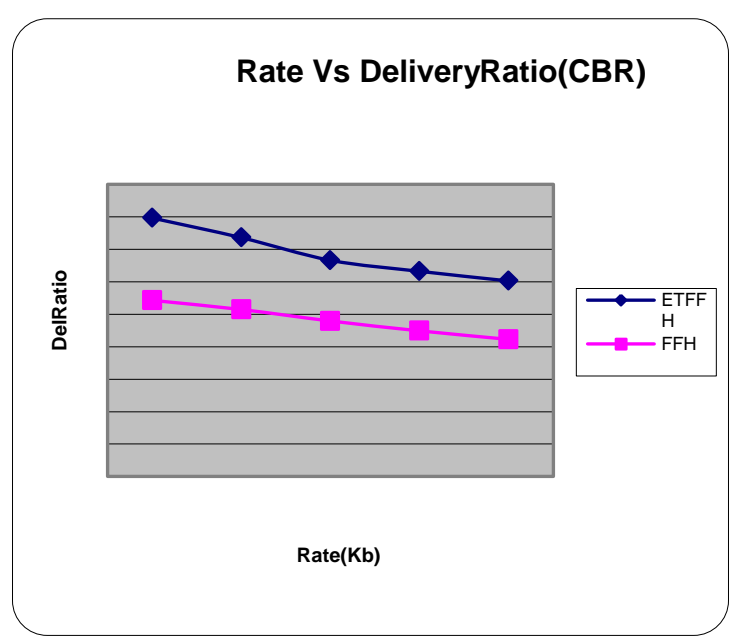

Fig. 3. Rate Vs Delivery Ratio

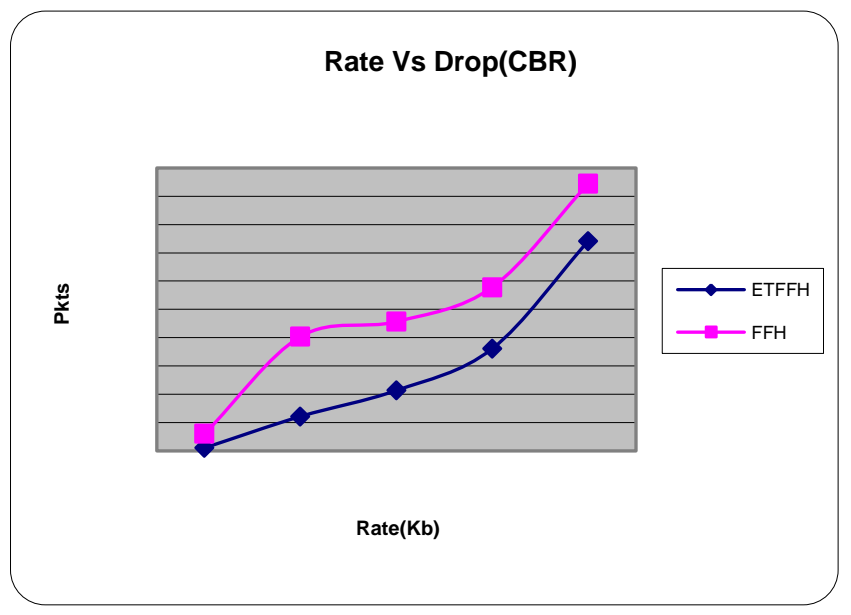

Fig. 4. Rate Vs Drop

Figures 2 to 4 show the results of delay, delivery ratio and packet drop for ETFFH and FFH when varying the rate of the CBR traffic flows. When comparing the performance of the two protocols, we infer that ETFFH outperforms FFH by $27 \%$ in terms of delay, $29 \%$ in terms of delivery ratio and $53 \%$ in terms of packet drop.

\section{Case-2 (EXP)}

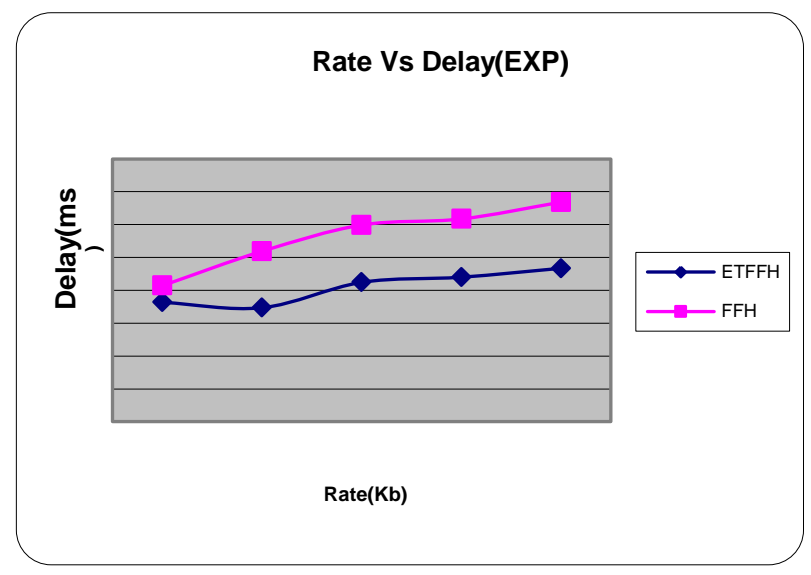

Fig. 5. Rate Vs Delay

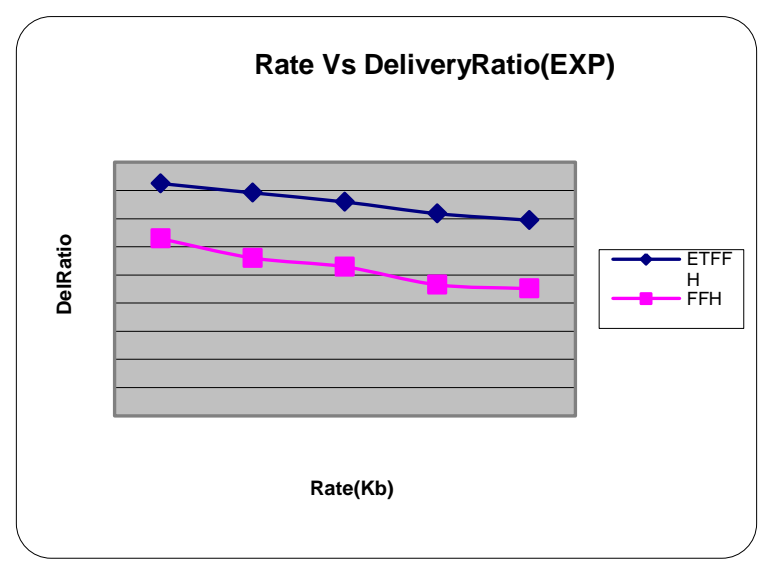

Fig. 6. Rate Vs Delivery Ratio

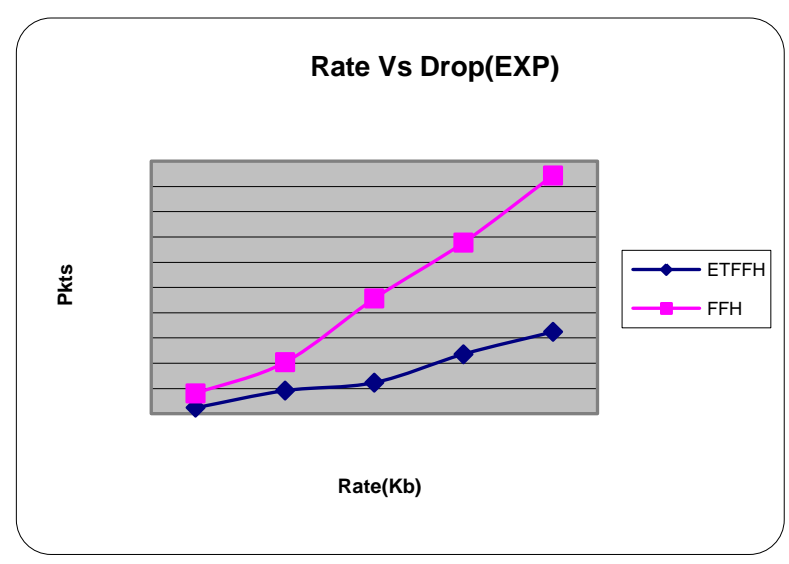

Fig. 7. Rate Vs Drop

Figures 5 to 7 show the results of delay, delivery ratio and packet drop for ETFFH and FFH when varying the rate of the EXP traffic flows. When comparing the performance of the two protocols, we infer that ETFFH outperforms FFH by $26 \%$ in terms of delay, $30 \%$ in terms of delivery ratio and $65 \%$ in terms of packet drop.

2) Varying the Traffic Flows

In third experiment, we vary the number of combined CBR and EXP traffic flows from 1 to 5. 


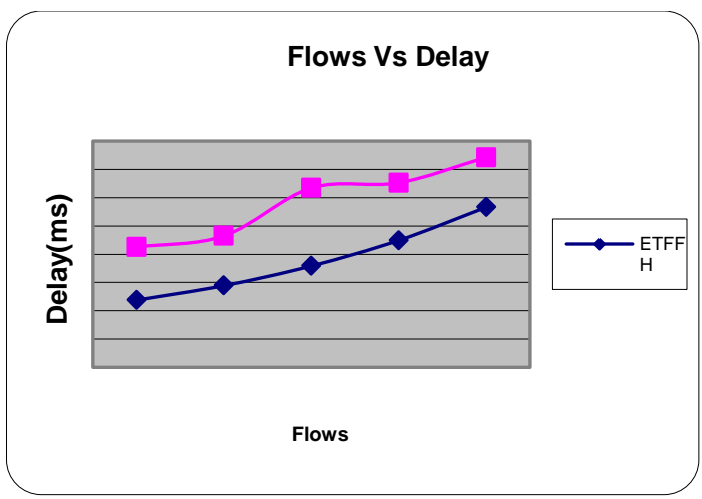

Fig. 8. Flows Vs Delay

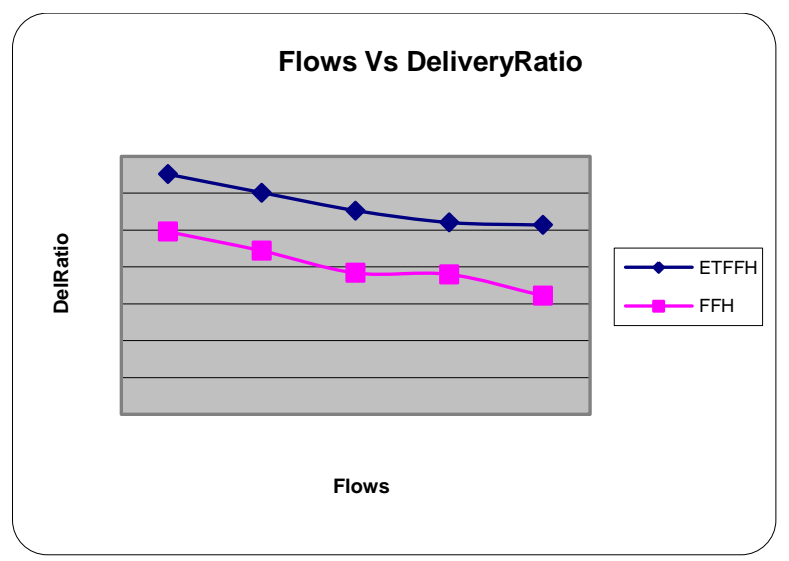

Fig. 9. Flows Vs Delivery Ratio

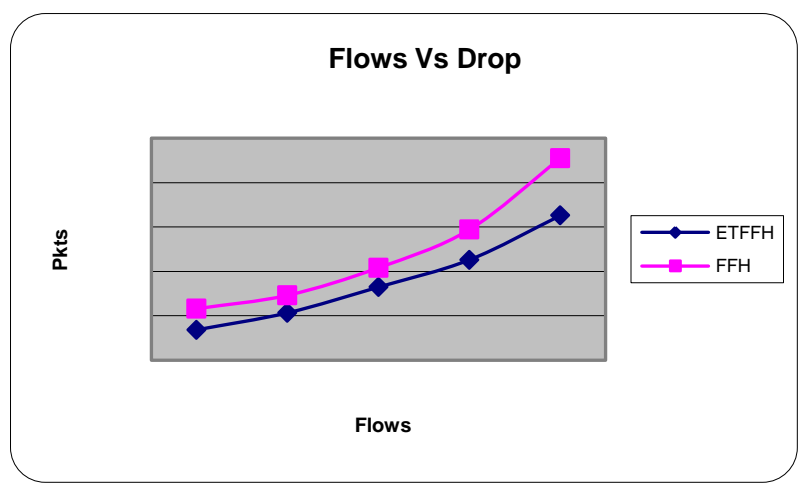

Fig. 10. Flows Vs Drop
Figures 8 to 10 show the results of delay, delivery ratio and packet drop for ETFFH and FFH by varying the flows. When comparing the performance of the two protocols, we infer that ACLBT outperforms MBLFGCP by $36 \%$ in terms of delay, $29 \%$ in terms of delivery ratio and $28 \%$ in terms of packet drop.

\section{CONCLUSION}

In this paper, we have proposed an Enhanced Tunneling Technique for Flow-based Fast Handover in Proxy Mobile IPv6 Networks. The proposed technique deals with the handoff operation being processed in Mobile IPv6 networks. This technique initially performs packet buffering prior handoff to ensure that during handoff packets are not lost. Next during the handoff process, the Flow Based Fast Handover technique is used. In this technique, the new subnet to which the mobile node is moving is regisstered at the Home Agent and at all the related routers. Then after verifying the connection, IPv6 tunnel is formed and traffic flow is redirected to the new subnet.

\section{REFERENCES}

[1] Mohit Bagde and P. Sankar, "IMPROVEMENT IN THE MOBILITY OF MOBILE IPV6 BASED MOBILE NETWORKS USING REVERSE ROUTING HEADER PROTOCOL AND FAST HANDOFF", International Journal of Mobile Network Communications \& Telematics ( IJMNCT) Vol. 3, No.6, December 2013.

[2] Miska Sulander, Timo H amalainen, Ari Viinikainen and Jani Puttonen, "Flow-Based Fast Handover Method for Mobile IPv6 Network", IEEE 59th Vehicular Technology Conference, VTC 2004-Spring, Volume:5, pp. $2447-2451$

[3] Yujin Noishiki, Yoshinori Kitatsuji, Hidetoshi Yokota, "Right-time Path Switching Method for Proxy Mobile IPv6 Route Optimization”, ICNS 2011 : The Seventh International Conference on Networking and Services, 2011.

[4] Hooshiar Zolfagharnasab, "Reducing Packet Overhead In Mobile IPv6", International Journal of Distributed and Parallel Systems (IJDPS) Vol.3, No.3, May 2012.

[5] Khaled Zeraoulia and Nadjib Badache," Seamless Multimedia Handoff for Hierarchical Mobile IPv6", The Seventh International Conference on Wireless and Mobile Communications, 2011

[6] Ali Safa Sadiq, Norsheila Binti Fisal, Kayhan Zrar Ghafoor and Jaime Lloret," Advanced Mobility Handover for Mobile IPv6 Based Wireless Networks", The Scientific World Journal, Volume 2014, Article ID 602808, 20 pages

[7] Mohit Bagde and P. Sankar," IMPROVEMENT IN THE MOBILITY OF MOBILE IPV6 BASED MOBILE NETWORKS USING REVERSE ROUTING HEADER PROTOCOL AND FAST HANDOFF", International Journal of Mobile Network Communications \& Telematics ( IJMNCT) Vol. 3, No.6, December 2013 